\title{
3D printed elastic mould granulation
}

Clint Okeyo ${ }^{1,2}$, Dewan F. Chowdhury ${ }^{1}$, Karmen Cheung $^{1}$ and Nejat Rahmanian ${ }^{2, *}$ n.rahmanian@bradford.ac.uk

${ }^{1}$ Nemaura Pharma Ltd., Advanced Technology Innovation Centre, 5 Oakwood Drive, Loughborough, Leicestershire, LE11 3QF, UK

${ }^{2}$ Chemical Engineering Department, Faculty of Engineering and Informatics, University of Bradford, Richmond Road, Bradford, BD7 1DP

*Corresponding author.

\begin{abstract}
In the pharmaceutical industry, enhanced process understanding resulting in superior control of product attributes, has the potential to save up to $20 \%$ of process engineering and product development costs during drug development. With the aim of achieving enhanced process understating, a novel approach for granulation of fine powders is presented. First, a mould with the desired particle shape and size is created using 3D printing followed by casting using elastomeric material. The formulation is prepared through wet massing and tested as a thin film on flat elastomeric membranes. The thin film itself can be a product but it also gives a good indication of coating performance before coating the patterned elastic membrane with the formulation i.e., 3D printed elastic mould granulation. Results show that following granulation and drying, granules of controlled size and shape (e.g. cubic and $500 \mu \mathrm{m}$ ), strength, friability and flowability can be formed. The method presented may allow for more robust process development in particle engineering.
\end{abstract}




\section{Keywords}

Continuous; granulation; elastic; moulding; thin-film

\section{Introduction}

Size enlargement process is defined as any process where small particles are agglomerated, compacted, or otherwise brought together into larger, relatively permanent masses in which the original particles can still be distinguished. The result is a somewhat porous and irregular structure of particles bonded together (Ge et al., 2018). In this study, the terms of size enlargement and granulation are used interchangeably. Size enlargement is often mechanistically modelled as three distinct rate processes which occur simultaneously: (1) wetting and nucleation, (2) coalescence and growth, and (3) attrition and breakage (Litster and Ennis, 2004). Granulation is employed to improve the properties of a powder mixture: enhanced flowability, dissolution, compression and compaction characteristics, uniformity of drug distribution, product appearance; inhibit segregation and dusting (Shinde et al., 2014; Cantor et al., 2008). Due to these favourable advantages, granulation is used to make granules in the range of $50 \mu \mathrm{m}$ to $50 \mathrm{~mm}$ in food, detergents (Rahmanian et al., 2016), catalysts, pesticides, plastics, fertilisers, metalliferous ores, nuclear fuels, ceramics, carbon black, cement kiln feeds and pharmaceuticals (Ennis et al., 2008; Capes and Darcovich, 1997).

Granulation is normally categories into two groups: wet and dry granulation. Wet granulation is conventional, at least in the pharmaceutical industry, where it is estimated to be used for approximately $80 \%$ of the cases (Shinde et al. 2014). Wet granulation is defined as modifying particle structure and morphology through the use of liquid acting on the powder blend to form inter-particulate bonds which then result in granules of varying sizes 
(Cantor et al., 2008). Wet granulation technologies include high shear, low shear (Cantor et al., 2008), fluidised bed (Passerini et al., 2010) and twin screw extrusion (Khorsheed et al., 2018). The advantages of wet granulation include a versatility in formulations, reduced binder quantity, short processing times, ability to process highly cohesive materials, predictable end point determination to list a few (Cantor et al., 2008). Disadvantages consists of and are not limited to a significant loss of material during dispending and drying, labour intensive tasks, long drying times, difficulty in scale up and a lack of mechanistic understanding despite years of experience (Suresh et al., 2017).

Dry granulation is an unconventional technology that can be subdivided into two categories: roller compaction and slugging (Guigon et al., 2007). Roller compaction is by far the most commonly used technique in dry granulation. It is desirable due to the absence of water which is attractive for drugs which are moisture/heat sensitive, its fitness for continuous processing, high drug loading (70-100\%) and flexibility in volume of production. The problems with roller compaction include a lack of mechanistic understanding, susceptibility of dusting, loss in compactability and limited applicability (Bindhumadhavan et al., 2005).

Alternative granulation technologies consist of: steam granulation, moisture activated dry granulation, pneumatic dry granulation, thermal adhesion granulation process, foamed binder technologies and freeze granulation technology (Shinde et al., 2014; Shanmugam, 2015). Generally the new technologies are able to produce granules with a good reproducibility in shorter processing times. They can also be environmentally friendly with few variables to optimise and lower operating costs. However, there are some recurring problems such as lack of holistic process understanding, high risk exposure to potent API, 
high capital costs and large equipment foot-print. There are also new challenges such as a lack of regulatory framework (Shinde et al., 2014; Shanmugam, 2015).

Additive manufacturing, also known as 3D printing, has recently been gaining traction in research and development in a wide variety industries and process technologies like granulation (Bandyopadhyay and Bose, 2015; Chua and Leong, 2015; Ge et al., 2017,2018). This is because the patents recently expired in circa 2010 allowing hobbyists and the like to take full advantage of 3D printing's extremely high flexibility in design e.g., unique 3D netshape with high strength-to-weight ratio (Thompson, 2007). Some key challenges 3D printing has yet to overcome include high capital cost and relatively long operating times for high volume manufacture (Bandyopadhyay and Bose, 2015). In this work it is used for prototyping, as is normally the case (Thompson, 2007), but recently 3D printing has been applied to granulation (Ge et al., 2017,2018) and other solid and/or liquid oral dosage forms (Genina et al., 2017). Ge et al. $(2017,2018)$ were able to use 3D printing to substantially improve current knowledge on the third rate process, attrition and breakage.

One of the strong points about the work by Ge et al. $(2017,2018)$ is that the granules have consistent shape and properties so more conclusive conclusions can be made based on these features. As a 3D printing technology method, the Ge et al. $(2017,2018)$ method suffers from the common challenge of high volume production. As far as the authors are aware, no granulation methods have been presented where prototype granules can be made before transferring to mature technologies like polymer injection moulding and extrusion where high volume manufacture can be achieved. This is because the produced granules are normally too complex for transfer to other process technologies. 
Nemaura Pharma Ltd.'s (henceforth: NPL) process for granulation is very different compared to the other processes presented. The process is also known as 3D printed elastic mould granulation (3DEMG). NPL's manufacturing method consists of the formation of a film on a substrate and then stretching the substrate along one or more axes to fracture the film into particle structures. The substrate may be moved continuously along a production line as the film is formed followed by stretching through acceleration. The substrate may contain elevated structures patterned in such a manner as to control the fracturing of the film (Chowdhury, 2015).

NPLs process may be much more capable in producing granules that are within size specification and may be similar enough to current granules to produce on pilot or full industrial scale within a relatively short timescale. The aim of this work is to produce and control the properties of granules by producing them using 3DEMG. This is done by producing granules using 3DEMG followed by characterisation of critical granule properties.

\section{Experimental}

\subsection{Materials}

Ibuprofen Grade 40 supplied by SI Group (Schenectady, USA) was used as the API. The bulk density was $0.33 \mathrm{~g} / \mathrm{mL}$. As stated in the European Medicines Agency (EMA) guidelines, purified water is to be used as the liquid medium (Gohlke and Doke, 2014). Aqueous solutions of sugars and synthetic polymers were used as the binding agents: sucrose (Fisher Scientific, UK), anhydrous-glucose, fructose (Acros Organics, USA), sorbitol (Calbiochem, USA), polyvinyl pyrrolidone K90 (PVP) (VWR, UK), polyethylene glycol 4000 (PEG) (Sigma Aldrich, UK). Non-ionic surfactants were also used as wetting agents: Kolliphor P 407 (BASF, Germany), Tween 20 (Acros Organics, USA). Silicone adhesive and silicone oil for casting 
were both supplied by Techsil (Warks, UK). Silicone (manufactured by Silex, UK), parafilm (manufactured by Bermis, UK) and 3M 9776 polyethylene single sided foam medical tape (henceforth: pink foam) (manufactured by $3 \mathrm{M}$, UK) were used as substrates. A saline phosphate buffer (PBS) supplied by (AppliChem, Germany) was used as the disintegration/dissolution medium.

\subsection{Equipment}

The manual film applicator (manufactured by Gradco, USA) uses a knife head coating configuration. The films are dried using a $25 \mathrm{~L}$ vacutherm vacuum oven (manufactured by Thermo Scientific, UK). SolidWorks 2016 CAD software and Objet30 Prime (manufactured by Stratasys) were used for 3D printing. The substrates were stretched using Hoffmann type pinchcocks of $50 \mathrm{~mm}$ bandwidth (manufactured by VWR, UK). Tensometer (manufactured by Mecmesin, UK) was used to measure the strain energy of substrates and for strength testing. The tensile tester was fitted with an upper and lower punch with outer diameters of $13.5 \mathrm{~mm}$. Tensile grips of $10 \mathrm{~mm}$ width were also used for strain energy measurements. British standard sieves (manufactured by Endecotts, UK) and an Axioskope 40 optical microscope equipped with an Axiocam (manufactured by Zeiss, UK) were used for granule characterisation. A UC152D stir-hot plate (manufactured by Stuart, UK) was used for disintegration/dissolution testing.

\subsection{Methodology}

3DEMG is the combination of wet agglomeration (Hapgood and Smith, 2015) with a modified transdermal patch manufacturing process (Ghosh et al., 1997). In this case an elastic substrate is used in place of a rigid substrate. In this study 3DEMG is used to fabricate either films for sublingual/buccal administration or discrete granules of controlled size and 
shape for capsules/tablets. Part of the methodology involves analysis of formulation performance during film application because this is theorised to be linked to performance of 3DEMG. As shown later, the formulation of the substrate is identical but surface features differ. Therefore, for example, a consistently strong attachment of thin-film to substrate after stretching is hypothesised to result in strong attachment of formed granules to the substrate after stretching.

The desired granule size and shape is selected when designing the master mesh. As shown in Figure 3, the master mesh is designed on SolidWorks. The mesh in Figure 1 shows that the desired particle size and shape can be $500 \mu \mathrm{m}$ and cubic while Figure 2 shows that the desired particle size and shape can be cuboid. Figure 4 shows that the mesh is then printed using the Objet30 Prime. Figure 5 shows that to obtain the elastic polymer membrane, silicone adhesive and silicone oil are casted onto the master meshes in a ratio of 10:1 (adhesive: oil) with the maximum substrate thickness being limited by the height of the mesh.

The first step of 3DEMG is to mix the API with excipients. This can be done in a batch, semibatch or continuous set-up. In this study the mixing was done in a batch set-up using a scintillation vial and spatula. The Ibuprofen was passed through a $1 \mathrm{~mm}$ sieve before mixing. Ibuprofen was selected as a model compound as the crystals are needle-like exhibiting poor flowability (i.e. cohesive) (Qu et al., 2017), brittle fracture, dusting, segregation and a tendency of lumping together (Le et al., 2006). Therefore, the effectiveness of 3DEMG can be assessed. During the mixing, unless otherwise stated, the composition of each mixture is given in Table 1. A liquid content of $75 \%$ was chosen for wet massing as it was deemed an adequate amount for ease of application of the formulation on the substrates while 
maintain a satisfactory drug loading of $25 \%$ API. It is important to note that when the sugar solutions were combined with the API to form slurries/pastes they showed characteristics similar to pseudoplastic fluids (Chhabra, and Richardson, 2011).

After mixing, the formulation is then placed on the substrate followed by application or alternatively, application and mould filling using the film applicator left-to-right (LTR). The landing areas on the substrate are then cleaned off using the film applicator right-to-left (RTL). The formulation is dried using the vacuum oven forced convection set-up - vacuum pump is turned-on and the air-inlet is left slightly open. To separate the granules from the substrate, the substrate is stretched to $30 \%$ elongation using Hoffmann pinchcocks and released instantly. As a result of the stored strain energy being released instantly, the granules are dislodged from the mould. The separation is conducted in a closed chamber fitted with a looking glass in order to contain the propelled granules.

As implied earlier, a consistently strong attachment of thin-film to substrate after stretching is hypothesised to result in poor solid-solid separation of formed granules after stretching. Consequently, similar to what is shown in Table 2, a number of binders are tested at different solid concentrations and film thicknesses before selecting a binder for granulation. As an example, the formulations which performed well were tested up to 9 times for sorbitol $60 \%$ solution and 4 times for PVP $20 \%$ solution.

\subsubsection{Granule characterisation}

The granule size distribution has been characterised by sieve analysis. A series of 3 British Standard (BS) test sieves $(200,400,1000 \mu \mathrm{m})$ were stacked and vibrated manually for 5 minutes. Granule shape was characterised by optical microscopy. The friability was determined using one BS standard sieve of $1 \mathrm{~mm}$ for granules greater than $1 \mathrm{~mm}, 0.4 \mathrm{~mm}$ 
for $1.0 \mathrm{~mm}$ granules and $0.2 \mathrm{~mm}$ for $0.5 \mathrm{~mm}$ granules; following the method presented by Birch and Marziano (2013) using two minutes per cycle. Three experimental runs per granule size were done for sieving and a single run was done per granule size for friability.

Flowability measurements were done using the angle of repose method of Copley Scientific (2016) which, with the exception of the protruding outer lip, adheres to European Pharmacopoeia Chapter 2.9.36 and US Pharmacopeia Chapter $<1174>$ on Powder Flow. The base had a fixed diameter of $23.5 \mathrm{~mm}$. Each flowability measurement was taken three times.

The granule strength has been characterised by the quasi-static side crushing test method (Rahmanian and Ghadiri, 2013). The individual granules are compressed between two punches using the tensile testing machine. In order to obtain some statistics, at least 10 granules were tested per sample. The exact procedure for this method is described by ASTM Standard D4179 (1982), Ryu and Saito (1991) and Couroyer et al. (2000). Typical forcedisplacement data when agglomerates break have been presented by Walker et al. (1997).

The tensometer was also used for measurement of silicone substrate strain energies. A cut out of $80 \mathrm{~mm}$ by $10 \mathrm{~mm}$ samples of substrates are clipped onto the tensometer. They are then stretched at the same set rate of $10 \mathrm{~mm} / \mathrm{min}$ until $30 \%$ elongation is reached. As illustrated in Equation 1 (Hamper, 2009), this produces a straight-line force-displacement curve that can be used to calculate the strain energy.

The bioavailability of a drug is determined by its dissolution over time. Before dissolution of the drug occurs, the granule and/or tablet must undergo wetting and disintegration (Smrčka et al.,2016). Disintegration testing was done by placing $20 \mathrm{mg}$ granules per $4 \mathrm{~g}$ PBS solution of $\mathrm{pH}$ 7.4. Disintegration end-point of the starting material was done by visual inspection. 


$$
E=1 / 2 F x=1 / 2 k x^{2}
$$

Where, $E$ is elastic strain-energy, $F$ is force, $x$ is displacement and $k$ is the stiffness constant.

\section{Results and discussion}

\subsection{Formulation studies}

\subsubsection{Effect of binder selection}

The studied sugars included sucrose, glucose, fructose and sorbitol. As shown in Figures 6 to 9, all sugars appear to exhibit an acceptable level of adherence to the silicone substrate during the application as formed films had an aspect ratio (width/length) much greater than 1/15. The synthetic polymers included PEG and PVP. In the selection of binders health and safety is the vital consideration. The sugars are usually cariogenic except for sorbitol in this case, while the polymers are essentially non-toxic as they are not absorbed in the gastrointestinal tract or mucous membranes (Rowe et al.,2013; Hartwig, 2013). PEGs are practically not volatile so PEG 4000 formulations were not studied further (Hartwig, 2013). For PVP in order to adhere to the chosen substrates the binder content tends to be much higher than the usual and processing of such viscous solutions is cost intensive. Therefore, sugars are probably more suitable for the chosen process.

Figure 6 illustrates that the films formed using fructose solutions have an aspect ratio much greater than $1 / 15$ but were unable to dry in a reasonable time (less than 18 hours). Figure 7 displays films formed using glucose which, upon drying, stuck onto the substrate strongly and this is unsuitable for processing. Figure 8a shows films formed using sucrose which dry in in less than 18 hours while undergoing crystallisation during solidification. Figure $8 \mathrm{~b}$ shows that when dried, the films underwent multiples fractures but remained attached to the substrate so solid-solid separation becomes challenging. On the other hand, films 
formed using sorbitol, as shown in Figure 9, are much easier to remove from the substrate. Like sucrose films, they also dry in less than 18 hours and undergo multiple fractures. In the light of these results, sorbitol formulations were used for the study.

\subsubsection{Effect of binder concentration}

The binder concentration affects the process performance in a number of ways. A generally high binder content leads to creating a more cohesive network of all ingredients (see Figures 10a, 11a and 12a) (Cantor et al., 2008). Low binder concentrations appear to be undesirable where binders are the only excipient. For PVP, it is clear from Figure 10b that during film application the formulation displays a poor adherence to the foam substrate. Figure 14 shows a low binder concentrations of a sugar ( $40 \%$ wt sucrose) which lead to a significant increase in drying times greater than 18 hours. Figures $12 b, c$ and $13 a$ show high binder concentrations (30 \%wt PVP) also appear to be undesirable as inconsistent films are more likely to be produced.

Figure $8 \mathrm{~b}$ (60\%wt sucrose) and Figure 11b (20\%wt PVP) show that there seems to be an optimum binder concentration where suitable adherence to substrate, consistent film thickness and acceptable drying times are observed. As an increased binder concentration leads to enhanced binding it was important to study whether disintegration of the films was still possible. In the case of PVP, this was not a concern as it is usually used to enhance the dissolution of poorly soluble drugs (Rowe et al., 2013). As highlighted in Table 6, it was determined that the disintegration generally occurs in less than 5 minutes. Maintaining a short disintegration time of less than 5 minutes for high binder concentrations, is desirable as it may lead to high strength granules and tablets that are able to disintegrate/dissolve as wanted. 


\subsubsection{Effect of surfactant addition}

Surfactants may act as detergents, wetting agents, emulsifiers, foaming agents, and dispersants (Porter and Porter, 1991). In this case non-ionic surfactants were used as wetting agents so that a lower concentration of binder may be used. As shown in Figures 15 and 16 the adherence to substrate was modified by the use of Tween 20 but drying of the films did not occur in less than 18 hours. Tween 20 is liquid in standard conditions so this is to be expected. When the poloxamer P 407 was used foaming of the formulation occurred which is unwanted for film application i.e., poor control of film and probably granule properties.

\subsubsection{Effect of silicone casting composition}

Two formulations were tested. One with high silicone oil content (10:1 of silicone adhesive: silicone oil) and one with low silicone oil content (20:1 of silicone adhesive: silicone oil). It was found that high silicone oil content led to a more rigid material so this meant that for a given strain, more strain energy is stored for separation of the granules from the substrate. In addition, silicone moulds made using the 20:1 ratio are able to remain rigid during the mould filling process, maintaining the desired structure of the formulation being casted. However, Figure 20 and Equation 1 support the decision of selecting the 10:1 ratio as the elastic mould becomes stiffer with increasing thickness. Therefore a thick substrate with 20:1 silicone adhesive-to-oil ratio may be too stiff to stretch to $30 \%$ elongation.

\section{2 Process studies}

\subsubsection{Effect of substrate material properties}

Both of the materials used for this study, silicone and parafilm, are hydrophobic. Parafilm is especially hydrophobic having a contact angle of $103^{\circ} \pm 11^{\circ}$ with water and a RMS roughness 
of $\sim 60\left(50 \times 50 \mu \mathrm{m}^{2}\right)$ as presented by Yamaguchi et al. (2016) and Ping et al. (2009). In the case of silicone (polysiloxane), it is generally hydrophobic having a high contact angle with water. However, silicone can undergo surface treatment/hydrophilisation to increase its wettability with water (Roth et al., 2008). In this study the silicone did not undergo chemical treatment.

When stretched, parafilm does not return to its original length while silicone quickly returns to its original after release. This means that the energy is dissipated during elongation for parafilm while it is mainly stored as strain energy for silicone. Therefore, parafilm would be unsuitable for a continuous manufacturing process. Furthermore, as shown in Figures $11 \mathrm{~b}$ and 17 , due to parafilms surface properties the ability of a formulation to adhere on the substrate homogeneously is poorer than with silicone. While the film of the same formulation contracts inwardly for parafilm (see Figure 17), it effectively coats silicone (see Figure 11b).

As shown in Figures $10 \mathrm{~b}$ and $12 \mathrm{c}$ there are cases where pink foam was coated with silicone to create a substrate with the combination of two elastic constants. This was to prevent the applied formulation from leaking through the pink foam. Solid-solid separation would probably be different as the strain energy storage and dissipation would probably be different for pink foam-silicone as compared with the pure silicone. Further studies are required to investigate this in the future.

\subsubsection{Effect of film thickness and substrate thickness}

The film thickness of the PVP formulation is $500 \mu \mathrm{m}$ in Figure 11 and $200 \mu \mathrm{m}$ in Figure 18. In Figure 11 , when it is stretched to $30 \%$ elongation, the film is delaminated from the substrate while multiple fractures occur in Figure 18. The same phenomena occurred with the sorbitol 
formulation. Figure 19 shows that at a thickness of $300 \mu \mathrm{m}$ it delaminated while Figure 9c shows that at a film thickness of $100 \mu \mathrm{m}$ multiple fractures occurred. Hence, there may be a critical film thickness/range of film thickness values for which either multiple fractures or delamination occurs. The distinction between multiple fractures and delamination is probably also a function of solid-liquid content.

The parafilm membrane thickness was always $127 \mu \mathrm{m}$ as it was purchased from the vendor (Bermis, UK). On the other hand, the silicone was casted in house so the thickness of the polymer membrane could be varied. As expected, Figure 20 shows an increase in the thickness of a silicone membrane appears to result in a stiffness constant. The smallest thickness casted was $300 \mu \mathrm{m}$ while the largest thickness was $6.0 \mathrm{~mm}$.

\subsubsection{Effect of patterned surfaces}

By patterning an elastomer via moulding one can obtain granules of desired size and shape. In this study two types of surface/moulding patterns were explored: cuboid and cubic. In addition to the cubic granules, cuboid moulds were made to illustrate the versatility in size and shape control with elastic mould granulation. Figures 21 and $23 \mathrm{c}$ show cuboid granules of $0.5 \mathrm{~mm}, 1.0 \mathrm{~mm}$ and $5.0 \mathrm{~mm}$ width while, Figures 22, 23, 25 show cubic granules of 0.5 $\mathrm{mm}, 1.0 \mathrm{~mm}, 2.0 \mathrm{~mm}$ and $4.0 \mathrm{~mm}$ size. Figure 23 shows that if the landing areas on the surface of the substrates are not cleaned-off effectively, agglomeration of the granules may occur due to formation of solid bridges (Okeyo et al., 2017). It is postulated that offspecification size is owing to incomplete mould filling (Kalpakjian and Schmid, 2006) and/or agglomeration of granules during drying (Litster, 2016) due to incomplete land area cleaning. 
Figures 24 and 25 show that optical microscopy and sieving have been used to check that the granules produced are very similar to the size and shape of the elastic mould. The cubic shape was selected as it is easy to mould and it exhibits good flowability (Carr, 1965) using the categories from the US Pharmacopeia (see Table 3) (Hoag and Lim, 2016; Pharmacopeia, 2005). Brittle fracture is the most common failure mode for pharmaceutical granules but, for the tested sorbitol formulation, strength testing revealed that the granules appear to undergo relatively slow irreversible deformation. Figure 26 and 27 display trends similar to a force-displacement curve at the onset of plastic deformation as presented by Zhou and Qiu (2010). Plastic deformation is desirable as it leads to high strength tablets (see Table 4) (Shiraishi et al., 1994, 1995). However, it is important to note that the cuboid granules exhibit multiple fractures on the long axis (see Figure 21c) and slow irreversible deformation on compression.

Assuming perfect mixing, the granules are $36 \% w t$ Ibuprofen and $64 \% w t$ sorbitol. Ibuprofen is susceptible to brittle fracture but as it is not the majority of the formulation, the granules are more characteristic of sorbitol (Shariare, 2012). Li et al. (2013) show that sorbitol deforms plastically so it is safe to assume that this is why the granules are deforming slowly and irreversibly during compression. Figure 28 displays data from friability tests which show that in some cases a less than $1 \%$ wt of material is lost and this is desirable for inhibiting dust formation (see Table 5). The low Agglomerate Brittleness Index (ABI) values also indicate the granules are hard (Birch and Marziano, 2013). Disintegration tests displayed in Table 6 also showed that granules are able to disintegrate in less than 5 minutes despite their high strength. 
Another detail worth mentioning is that the drying time of patterned substrates is significantly longer than the drying time of thin films. This is most likely to due to the mass transfer limitations introduced by reduction in effective surface area in contact with air, and increase in thickness of the formulation. Succinctly, the drying step is currently the rate limiting step. Preliminary studies have shown that one may be able to significantly reduce the drying time by reducing the liquid content and increase the binder concentration of the formulation.

\subsubsection{Effect of stretching}

Stretching the substrate resulted in either fracture or delamination of thin films. However, in the case of patterned surfaces, it only separated the surface of the granules from the surface of the polymer membrane. For the granules to be fully separated from the substrate the stretched membrane had to be released instantly to propel the granules out of the substrate i.e., strain energy solid-solid separation. The effect of strain energy on solid-solid separation is presented in Figure 29. Excluding the anomalous point at $66 \mathrm{~mJ}$ of strain energy, there appears to be a positive correlation between strain energy and solid-solid separation. This was hypothesised to be the case. Similar to Figure 20, a wider range or more data point measurements of strain energy would have to be made before statistical regression is undertaken.

\section{Conclusion}

A novel approach for the formation of API loaded films and granules is presented in order to attend to much needed solutions to some of the existing problems in formulation engineering: structural control, process understanding and continuous processing. NPL's process offers a platform for small scale experiments, ease of scale-up and smaller 
equipment footprint owing to continuous processing, as well as particulate structural control to produce granules of controlled size, shape, strength, friability and flowability. It is also important to note that NPL's process is not a solution to all the problems as it is unsuitable for moisture sensitive APIs. Overall NPL's process has the potential to either replace traditional technologies or compete as an alternative. 


\section{References}

A.S.T.M. Standard D4179.1982, Single Pellet Crush Strength of Formed Catalyst Shapes. American Society for Testing Materials.

Bandyopadhyay, A. and Bose, S., 2015. Additive manufacturing. CRC Press.

Bindhumadhavan, G., Seville, J.P.K., Adams, M.J., Greenwood, R.W. and Fitzpatrick, S., 2005. Roll compaction of a pharmaceutical excipient: Experimental validation of rolling theory for granular solids. Chemical Engineering Science, 60(14), pp.3891-3897.

Birch, M. and Marziano, I., 2013. Understanding and avoidance of agglomeration during drying processes: A case study. Organic Process Research \& Development, 17(10), pp.13591366.

Cantor, S.L., Augsburger, L.L., Hoag, S.W. and Gerhardt, A., 2008. Pharmaceutical granulation processes, mechanism and the use of binders. Pharmaceutical dosage forms: tablets, 1, pp.261-302.

Capes, C.E. and Darcovich, K., 1997. Size enlargement. John Wiley \& Sons, Inc.

Carr, R.L., 1965. Evaluating flow properties of solids. Chem. Eng., 72, pp.163-168.

Chhabra, R.P. and Richardson, J.F., 2011. Non-Newtonian flow and applied rheology: engineering applications. Butterworth-Heinemann.

Chowdhury, D. F. H., 2015. Formation of Particle Structures. United States Patent and Trademark Office (USPTO) Patent number 20150359741.

Chua, C.K. and Leong, K.F., 2015. 3D PRINTING AND ADDITIVE MANUFACTURING: Principles and Applications. $5^{\text {th }}$ edn. World Scientific Publishing Co Inc. 
Copley Scientific, 2016. Quality Solutions for the Testing of Pharmaceuticals. [Brochure].

Ennis, B.J., Witt, W., Weinekötter, R., Sphar, D., Gommeran, E., Snow, R. H., Allen, T., Raymus, G. J., Litster, J. D., 2008. 'Solid-Solid Operations and Processing' in Perry's Chemical Engineers Handbook. McGraw-Hill Professional Publishing.

Ge, R., Ghadiri, M., Bonakdar, T. and Hapgood, K., 2017. 3D printed agglomerates for granule breakage tests. Powder Technology, 306, pp.103-112.

Ge, R., Ghadiri, M., Bonakdar, T., Zhou, Z., Larson, I. and Hapgood, K., 2018. Experimental study of the deformation and breakage of 3D printed agglomerates: Effects of packing density and inter-particle bond strength. Powder Technology, 340, pp.299-310.

Genina, N., Boetker, J., Löbmann, K., Rades, T., and Rantanen, J., 2017. 'Additive Manufacturing of Multicompartmental Pharmaceutical Products' in $8^{\text {th }}$ International Granulation Conference. University of Sheffield, 28-30 June 2017. [Online] Available at https://www.sheffield.ac.uk/polopoly fs/1.709452!/file/Oraltime.pdf $\quad$ (Accessed: 29 November 2017).

Ghosh, T.K., Pfister, W.R. and Yum, S.I., 1997. Transdermal and topical drug delivery systems. Informa Health Care.

Gohlke, J.M. and Doke, D., 2014. Encyclopedia of toxicology. Elsevier.

Guigon, P., Simon, O., Saleh, K., Bindhumadhavan, G., Adams, M.J. and Seville, J.P., 2007. Roll pressing. Handbook of Powder Technology, 11, pp.255-288.

Hamper, C., 2009. Pearson Baccalaureate: Higher Level Physics for the IB Diploma (Pearson International Baccalaureate Diploma: International Edition. Pearson Education 
Hapgood, K.P. and Smith, R.M., 2015. Wet Granulation and Mixing. Pharmaceutical Blending and Mixing, p.153.

Hartwig, A. ed., 2013. The MAK-collection for occupational health and safety(Vol. 27). John Wiley \& Sons.

Hoag, S. and Lim, H.P., 2016. Particle and powder bed properties. Pharmaceutical Dosage Forms: Tablets. Unit Operations and Mechanical Properties, 1, pp.17-73.

Kalpakjian, S. and Schmid, S.R., 2006. Manufacturing engineering and technology. $5^{\text {th }}$ edn. Jurong, Singapore: Pearson.

Khorsheed, B., Gabbott, I., Reynolds, G.K., Taylor, S.C., Roberts, R.J. and Salman, A.D., 2018. Twin-screw granulation: Understanding the mechanical properties from powder to tablets. Powder Technology.

Le, V.N.P., Leterme, P., Gayot, A. and Flament, M.P., 2006. Influence of granulation and compaction on the particle size of ibuprofen-development of a size analysis method. International journal of pharmaceutics, 321(1), pp.72-77.

Li, X.H., Zhao, L.J., Ruan, K.P., Feng, Y. and Ruan, K.F., 2013. The application of factor analysis to evaluate deforming behaviors of directly compressed powders. Powder technology, 247, pp.47-54.

Litster, J. and Ennis, B., 2004. The science and engineering of granulation processes. Dordrecht: Kluwer Academic Publishers.

Litster, J., 2016. Design and Processing of Particulate Products. Cambridge University Press. 
Okeyo, C., Mallet, F., Rahmanian, N. and Schäfer, F., 2017. 'Scale Down of Agitated Filter Drying of Active Pharmaceutical Ingredients' in $8^{\text {th }}$ International Granulation Conference. University of Sheffield, 28-30 June, Paper 56.

Passerini, N., Calogerà, G., Albertini, B. and Rodriguez, L., 2010. Melt granulation of pharmaceutical powders: a comparison of high-shear mixer and fluidised bed processes. International journal of pharmaceutics, 391(1), pp.177-186.

Pharmacopeia, U.S., 2005. USP 29-NF 24. Rockville, MD: USP.

Ping, C.C.W., Ivanova, N.A., Starov, V.M., Hilal, N. and Johnson, D., 2009. Spreading behaviour of aqueous trisiloxane solutions over hydrophobic polymer substrates. Colloid journal, 71(3), pp.391-396.

Porter, M.R. and Porter, M., 1991. Handbook of Surfactants. Published by Blackie \& Son (Glasgow and London), pp.116-178.

Qu, L., Stewart, P.J., Hapgood, K.P., Lakio, S., Morton, D.A. and Zhou, Q.T., 2017. Single-step coprocessing of cohesive powder via mechanical dry coating for direct tablet compression. Journal of pharmaceutical sciences, 106(1), pp.159-167.

Rahmanian N., Halmi M. H., Kong C. C., Patel R., Yusup S., Mujtaba I. M. (2016) An experimental investigation on seeded granulation of detergent powders. Chemical Engineering Transactions, 52, pp.1297-1302

Rahmanian, N. and Ghadiri, M., 2013. Strength and structure of granules produced in continuous granulators. Powder technology, 233, pp.227-233. 
Roth, J., Albrecht, V., Nitschke, M., Bellmann, C., Simon, F., Zschoche, S., Michel, S., Luhmann, C., Grundke, K. and Voit, B., 2008. Surface functionalization of silicone rubber for permanent adhesion improvement. Langmuir, 24(21), pp.12603-12611.

Rowe, R.C., Sheskey, P.J. and Quinn, M.E., 2013. Handbook of pharmaceutical excipients7th edition. Pharm. Dev. Technol., 18, p.544.

Shanmugam, S., 2015. Granulation techniques and technologies: recent progresses. Biolmpacts: $B I, 5(1)$, p.55.

Shariare, M.H., 2012. The rational design of drug crystals to facilitate particle size reduction. Investigation of crystallisation conditions and crystal properties to enable optimised particle processing and comminution (Doctoral dissertation, University of Bradford).

Shinde, N., Aloorkar, N., Kulkarni, A., Bangar, B., Sulake, S. and Kumbhar, P., 2014. Recent Advances in Granulation Techniques. Asian Journal of Research in Pharmaceutical Science, 4(1), pp.38-47.

Shiraishi, T., Kondo, S., Yuasa, H. And Kanaya, Y., 1994. Studies on the granulation process of granules for tableting with a high speed mixer. I. Physical properties of granules for tableting. Chemical and pharmaceutical bulletin, 42(4), pp.932-936.

Shiraishi, T., Sano, A., Kondo, S., Yuasa, H. And Kanaya, Y., 1995. Studies on the granulation process of granules for tableting with a high speed mixer. II. Influence of particle size of active substance on granulation. Chemical and pharmaceutical bulletin, 43(4), pp.654-659.

Smrčka, D., Dohnal, J. and Štěpánek, F., 2016. Dissolution and disintegration kinetics of highactive pharmaceutical granules produced at laboratory and manufacturing scale. European Journal of Pharmaceutics and Biopharmaceutics, 106, pp.107-116. 
Suresh, P., Sreedhar, I., Vaidhiswaran, R. and Venugopal, A., 2017. A comprehensive review on process and engineering aspects of pharmaceutical wet granulation. Chemical Engineering Journal, 328, pp.785-815.

Thompson, R., 2007. Manufacturing processes for design professionals. Thames \& Hudson.

University of Birmingham, 2017. EPSRC Centre for Doctoral Training in Formulation Engineering. Available at: http://www.birmingham.ac.uk/schools/chemicalengineering/postgraduate/eng-d/index.aspx (Accessed: 07 August 2017).

Walker, G.M., Magee, T.R.A., Holland, C.R., Ahmad, M.N., Fox, N. and Moffatt, N.A., 1997. Compression testing of granular NPK fertilizers. Nutrient Cycling in Agroecosystems, 48(3), pp.231-234.

Yamaguchi, M., Aoyama, T., Yamada, N. and Chibana, H., 2016. Quantitative measurement of hydrophilicity/hydrophobicity of the plasma-polymerized naphthalene film (Super Support Film) and other support films and grids in electron microscopy. Microscopy, 65(5), pp.444-450.

York, D., 2017. 'Engineering Formulated Products' in ChemEngDayUK2017. University of Birmingham, 27-28 March 2017. [Online] Available at https://static1.squarespace.com Accessed: 05 August 2017.

Zhou, D. and Qiu, Y., 2010. Understanding material properties in pharmaceutical product development and manufacturing: Powder flow and mechanical properties. Journal of Validation Technology, 16(2), p.65. 
Figure 1. Cubic desired particle design and size with (A) $500 \mu \mathrm{m}$ to $5000 \mu \mathrm{m}$ length (B) 500 $\mu \mathrm{m}$ to $5000 \mu \mathrm{m}$ length (C) $500 \mu \mathrm{m}$ to $5000 \mu \mathrm{m}$ length and (D) $1.5 \mathrm{~mm}$ to $15 \mathrm{~mm}$.

Figure 2. Cuboid desired particle design and size with (A) $500 \mu \mathrm{m}$ to $5000 \mu \mathrm{m}$ length and (B) $500 \mu \mathrm{m}$ to $5000 \mu \mathrm{m}$ length.

Figure 3.SolidWorks CAD model of (a) $500 \times 500 \times 500 \mu \mathrm{m}$ cubic pattern (b) $1 \times 1 \times 80 \mathrm{~mm}$ cuboid pattern both (a) and (b) are on $50 \times 80 \mathrm{~mm}$ master meshes.

Figure 4. 3D print of master meshes from Figure 1a (left) and $1 \mathrm{~b}$ (right).

Figure 5. Silicone moulds of master meshes from Figure 1a (left) and 1b (right).

Figure 6. Ibuprofen-fructose $70 \%$ solution.

Figure 7. Ibuprofen-glucose $40 \%$ solution (a) applied (b) stretched.

Figure 8. Ibuprofen-sucrose $60 \%$ solution (a) optical microscopy (b) applied (c) stretched.

Figure 9. Ibuprofen-sorbitol 60\% (a) optical microscopy (b) applied (c) stretched.

Figure 10. Ibuprofen-PVP $10 \%$ solution (a) optical microscopy (b) applied.

Figure 11. Ibuprofen-PVP 20\% solution (a) optical microscopy (b) applied (c) stretched.

Figure 12. Ibuprofen-PVP $30 \%$ solution.

Figure 13. Ibuprofen-sucrose $80 \%$ solution (a) applied (b) stretched.

Figure 14. Ibuprofen-sucrose $40 \%$ solution.

Figure 15. Ibuprofen (20.8\% wt)-PVP 2\% (62.5\% wt)-Tween 20 (16.7\% wt). 
Figure 16. Ibuprofen (19.2 \% wt)-PVP 10\% (57.7\% wt)-Tween 20 (23.1\% wt).

Figure 17. Ibuprofen-PVP $20 \%$ solution on flat parafilm (a) light coating (b) heavy coating.

Figure 18. Ibuprofen-PVP $20 \%$ solution $(200 \mu \mathrm{m})$ (a) applied film (b) subsequently stretched membrane and fractured film.

Figure 19. Ibuprofen-Sorbitol $60 \%$ Solution $(300 \mu \mathrm{m})$

Figure 20. Graph of effect of substrate thickness on strain energy.

Figure 21. Cuboid granules (a) $0.5 \mathrm{~mm}$ (b) $1 \mathrm{~mm}$ (c) $5.0 \mathrm{~mm}$.

Figure 22. Cubic granules $0.5 \mathrm{~mm}, 1.0 \mathrm{~mm}, 2.0 \mathrm{~mm}, 4.0 \mathrm{~mm}$ LTR.

Figure 23. Effect of cleaning (a), (b) without surface cleaning (c), (d) with surface cleaning.

Figure 24. Size distribution of cubic granules (a) $1000 \mu \mathrm{m}$ (b) $500 \mu \mathrm{m}$.

Figure 25. Optical microscopy of cubic granules (a) $1000 \mu \mathrm{m}$ (b) $500 \mu \mathrm{m}$.

Figure 26. Force-displacement graphs for ten $1000 \mu \mathrm{m}$ cubic granules of ibuprofen-sorbitol $60 \%$ solution.

Figure 27. Force-displacement graphs for ten $500 \mu \mathrm{m}$ cubic granules of ibuprofen-sorbitol $60 \%$ solution.

Figure 28. Friability testing.

Figure 29: Effect of strain energy on solid-solid separation. 
Table 1. Formulation composition for mixing.

\begin{tabular}{|c|c|c|c|}
\hline Compound & Mass (g) & \% Weight & Comments \\
\hline Ibuprofen & 0.5 & 25 & \\
\hline Binder Solution & 1.5 & 75 & See Table 2 \\
\hline Total & 2.0 & 100 & \\
\hline
\end{tabular}


Table 2. Formulation composition for preparation of binder solutions (example).

\begin{tabular}{|c|c|c|c|}
\hline Compound & Mass (g) & \% Weight & Comments \\
\hline Sorbitol Binder & 6.0 & 60 & $\begin{array}{c}\text { Heat up to } 80^{\circ} \mathrm{C} \text { and } \\
\text { hold for } 30 \text { min while } \\
\text { agitating at } 3 / 9\end{array}$ \\
\hline Purified water & 4.0 & 40 & 100 \\
\hline Total & 10.0 & \multicolumn{2}{|c|}{} \\
\hline
\end{tabular}


Table 3. Effect of 3DEMG on flowability of granules.

\begin{tabular}{|c|c|c|}
\hline Compound & Angle of Repose $\left(^{\circ}\right)$ & Comments \\
\hline Ibuprofen Grade 40 & $63 \pm 1$ & Very Poor Flowability \\
\hline $1000 \mu \mathrm{m}$ Cubic Granules & $31 \pm 4$ & Good Flowability \\
\hline $500 \mu \mathrm{m}$ Cubic Granules & $42 \pm 4$ & Passable - may hang up \\
\hline
\end{tabular}


Table 4. Maximum normal stress experienced by granules.

\begin{tabular}{|c|c|c|}
\hline Compound & Normal Stress (MPa) & Equation \\
\hline $500 \mu \mathrm{m}$ Cubic Granules & 160 & \multirow{2}{*}{ Normal Stress = Force/Area } \\
\cline { 1 - 2 } $1000 \mu \mathrm{m}$ Cubic Granules & 40 & \\
\hline $5000 \mu \mathrm{m}$ Cubic Granules & 1.6 & \\
\hline
\end{tabular}


Table 5. Friability risk categories. (Birch and Marziano, 2013)

\begin{tabular}{|c|c|c|}
\hline Extent of Structural Integrity & Classification & Recommendation \\
\hline$<10 \%$ Mass Loss & Low & Acceptable formulation \\
\hline $10 \%<$ Mass Loss $<20 \%$ & Intermediate & Slight or no modification \\
\hline Mass Loss $>20 \%$ & High & Change formulation \\
\hline Agglomerate Brittleness Index & Classification & Recommendation \\
\hline$<0.1 \mathrm{ABI}$ or $\mathrm{ABI}=0$ & Low & Acceptable formulation \\
\hline $0.1<\mathrm{ABI}<1.0$ & Intermediate & Slight or no modification \\
\hline $\mathrm{ABI}>1.0$ & High & Increase binder content \\
\hline $\mathrm{ABI}>>1^{*}$ & Very high & Change formulation \\
\hline
\end{tabular}

${ }^{*} A B I$ $>1$ when no granules on sieve and $A B I=0$ when there is zero dust formation 
Table 6. Disintegration time results and categorical responses.

\begin{tabular}{|c|c|c|c|}
\hline Compound & $\begin{array}{c}\text { Time to } \\
\text { Disintegrate }(\mathrm{min})\end{array}$ & $\begin{array}{c}\text { Categorical } \\
\text { Response }\end{array}$ & Remarks \\
\hline Ibuprofen Grade 40 & 30 & More than 5 min & Dispersion of lumps \\
\hline Ibuprofen-PVP $25 \%$ sol. & 5 & Less than 5 min & Film disintegration \\
\hline Ibuprofen-sorbitol $60 \%$ sol. & 5 & Less than 5 min & Granule disintegration \\
\hline
\end{tabular}




\section{Highlights}

- A novel approach for structural control of particulates is presented.

- Effectiveness of process for granulation was evaluated.

- Exceptionally narrow granule size distribution is achieved.

- Granules exhibit slow irreversible deformation, low friability, good flowability and ease of disintegration.

\section{Graphical abstract}

From CAD model (a) to 3D Print (b), silicone mould (c), granulation (d), and characterisation (e) 


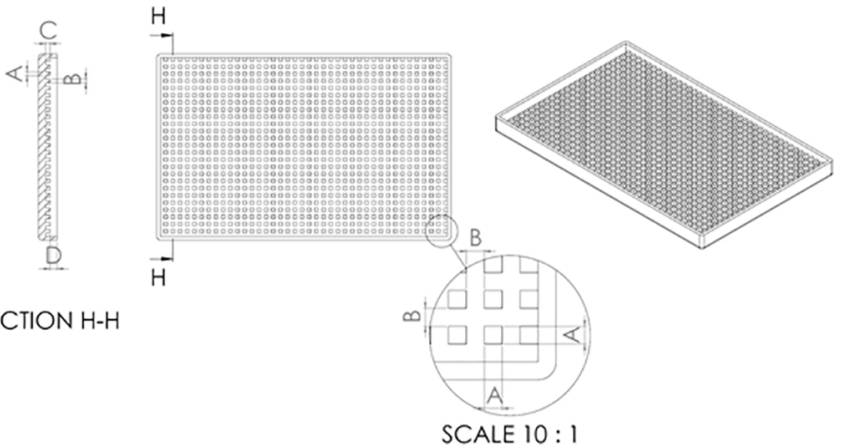

Figure 1 


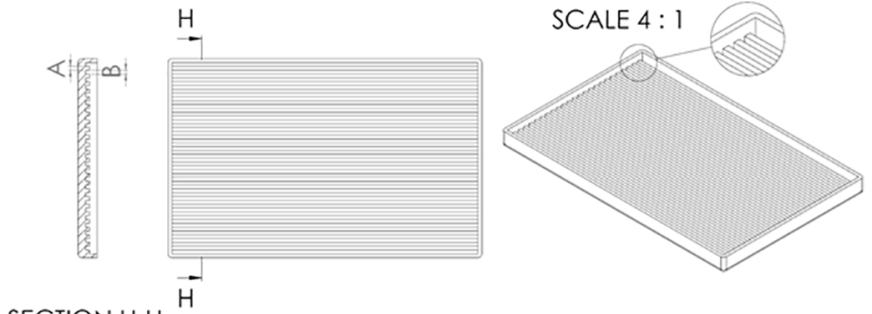

SECTION H-H

Figure 2 


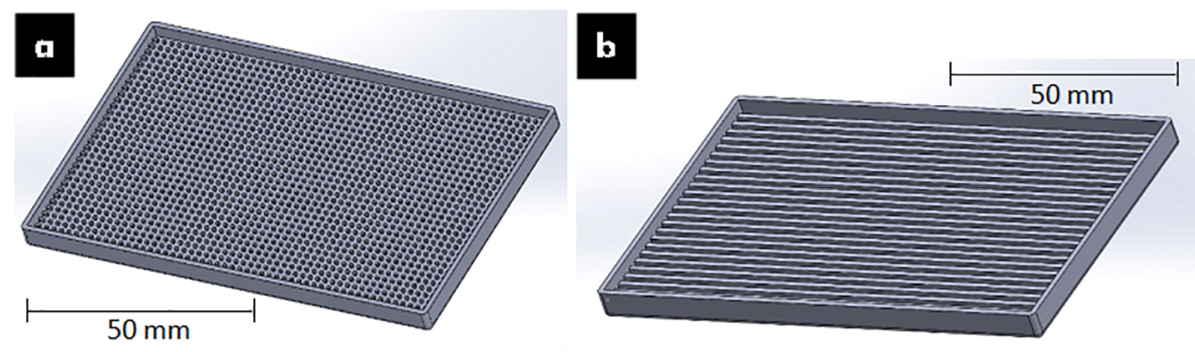

Figure 3 


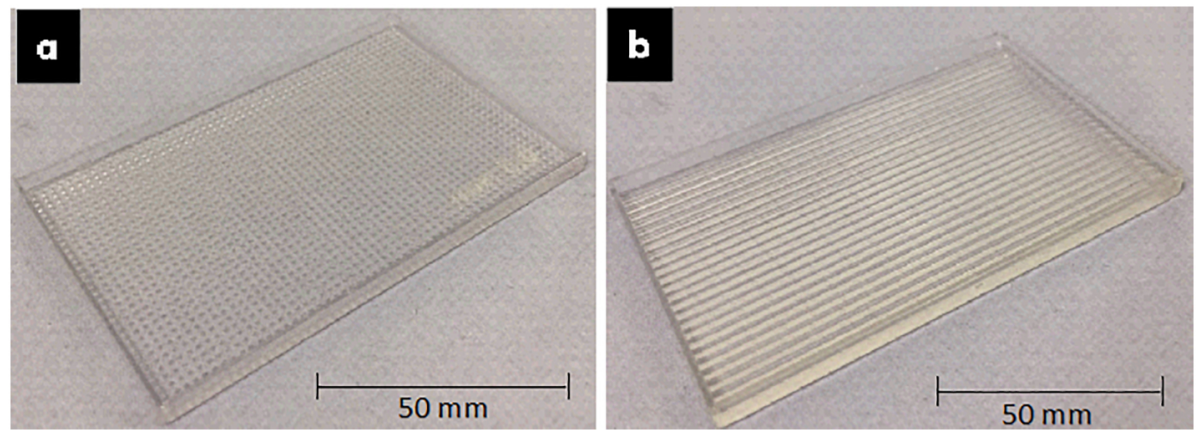

Figure 4 


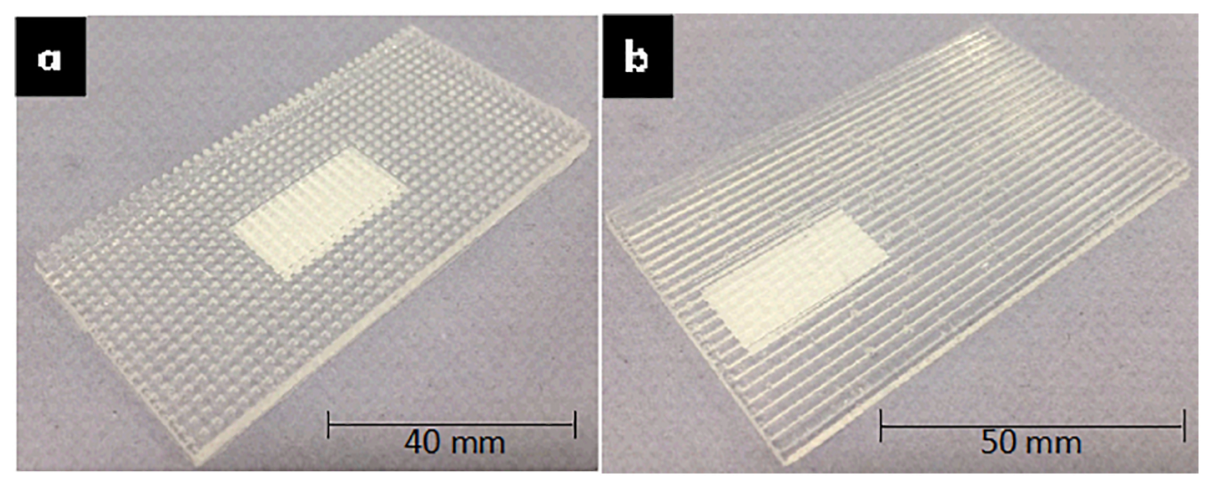

Figure 5 
Figure 6 

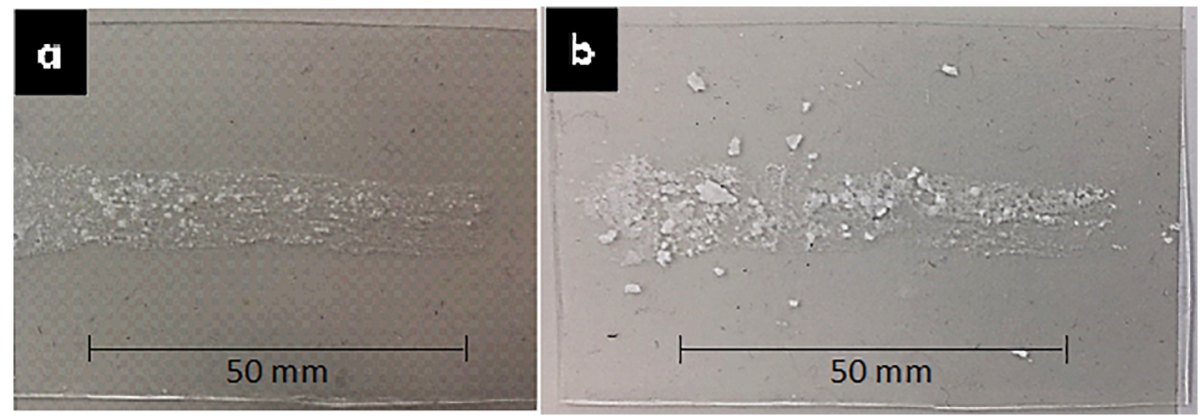

Figure 7 


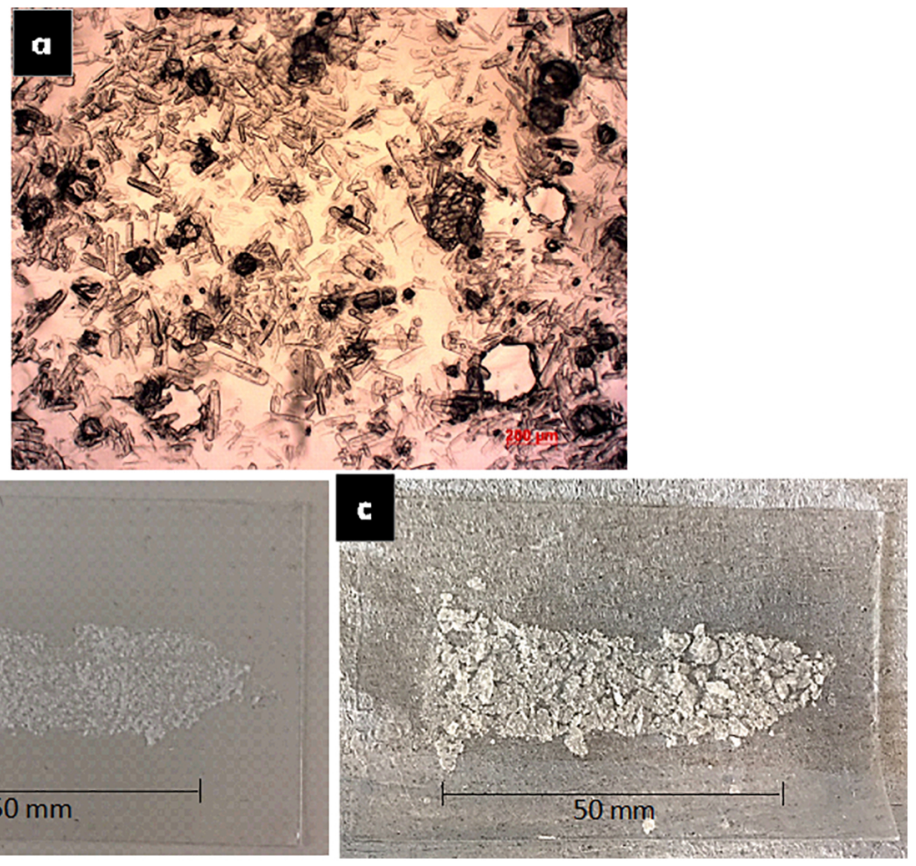

Figure 8 

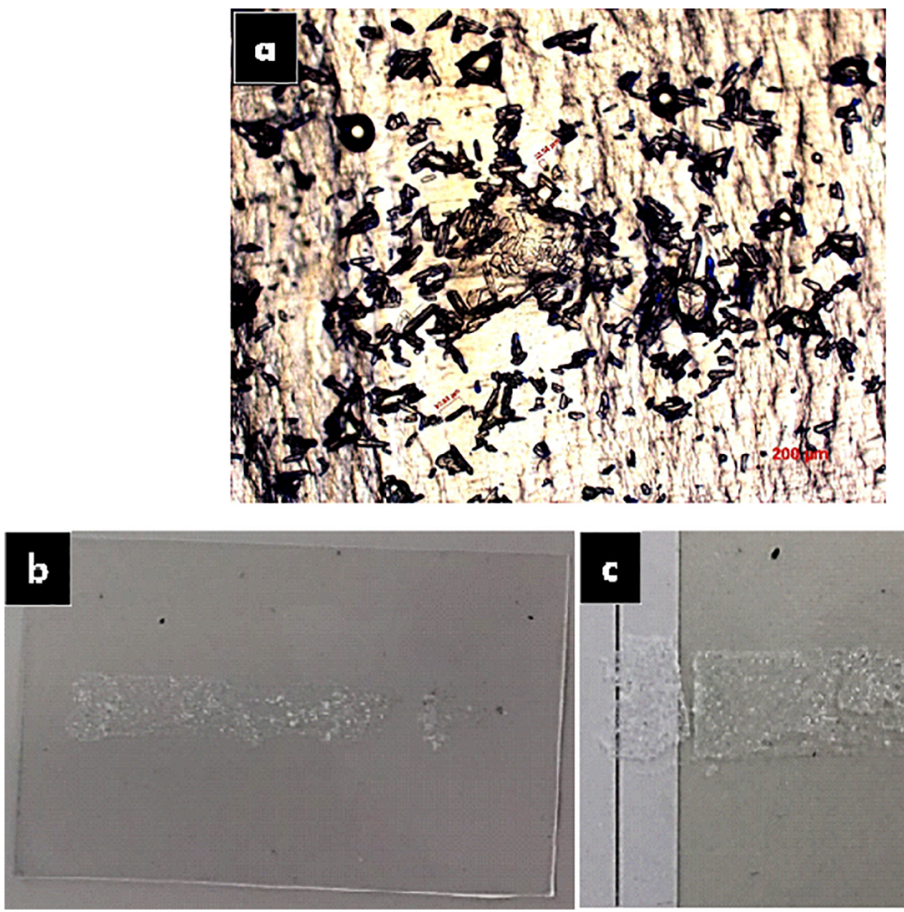

Figure 9 


\section{a}

1.

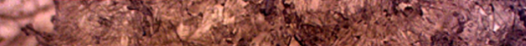

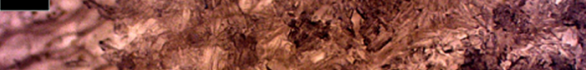

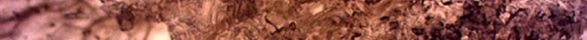

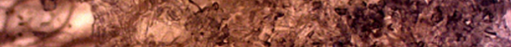

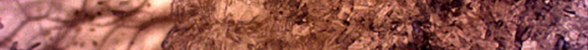

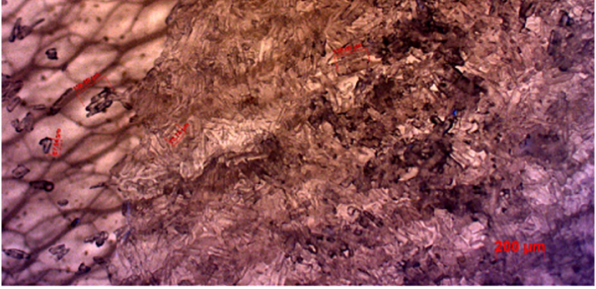

\section{b}

$50 \mathrm{~mm}$

Figure 10 


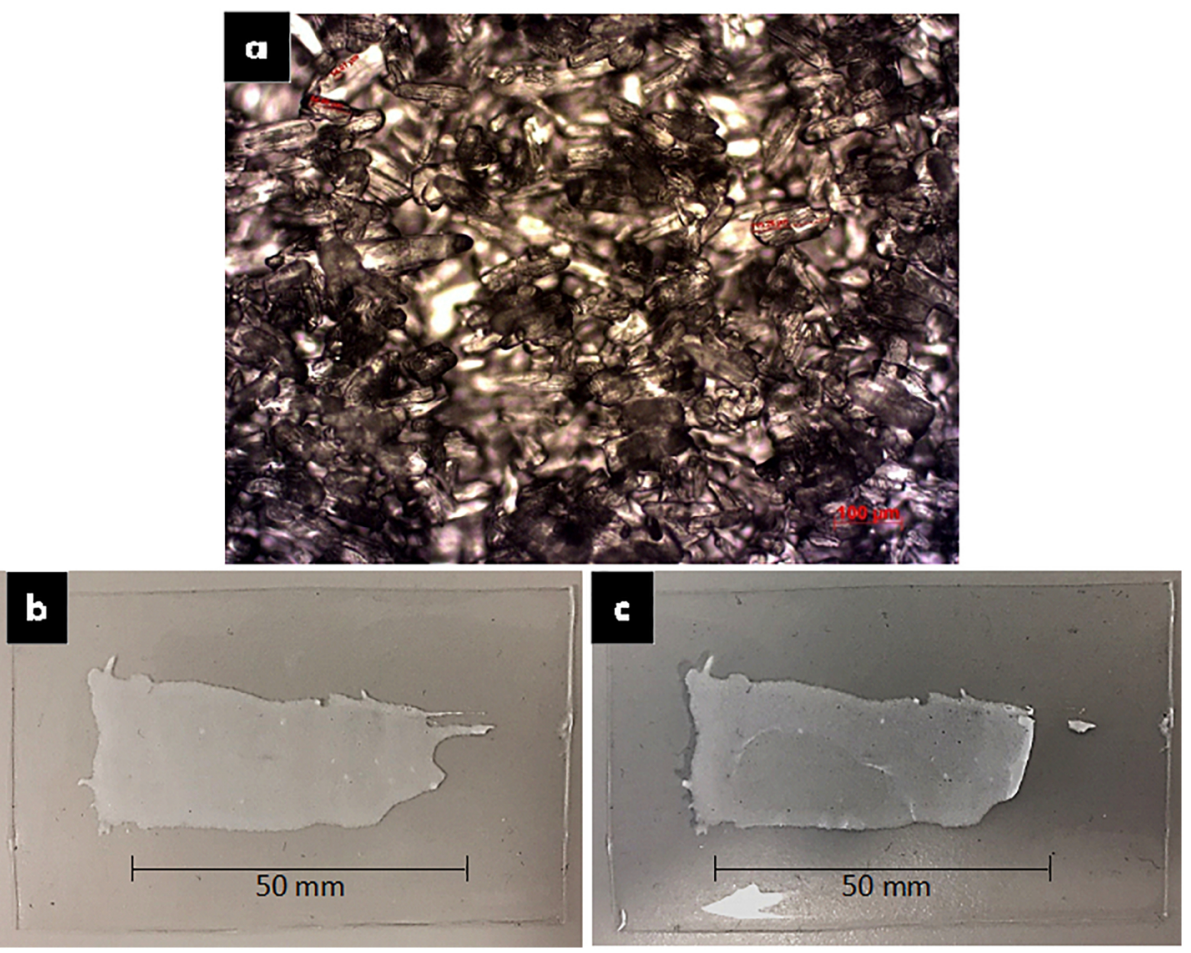

Figure 11 
- 10 20 $000 \%$ \%

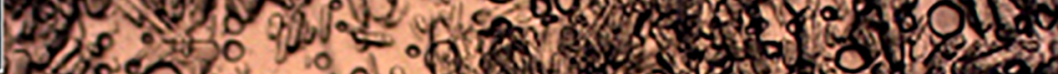
स.

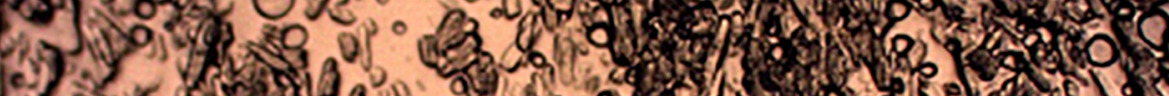

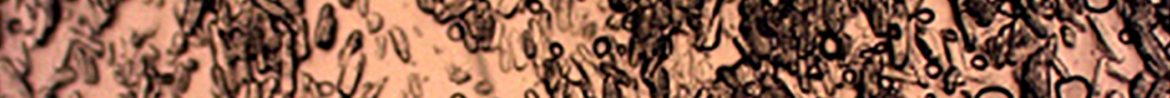

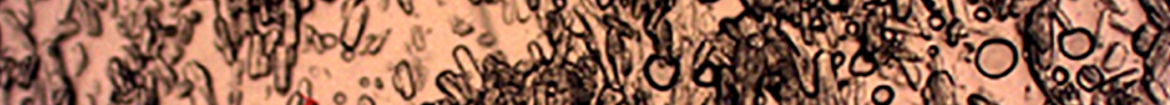

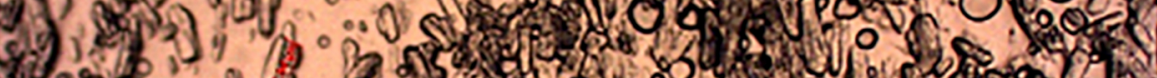

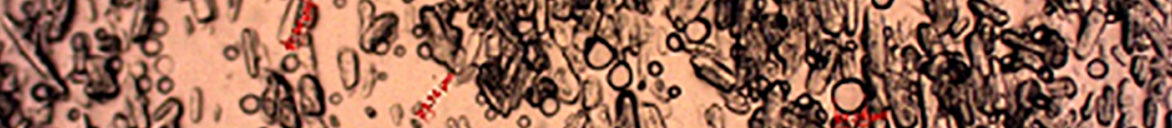
9.

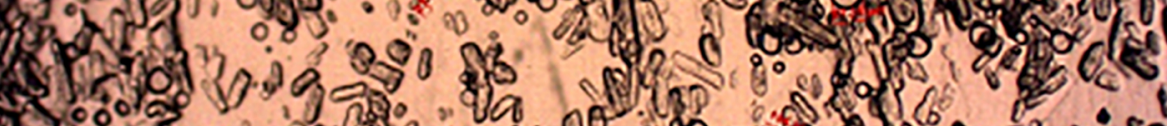
310 . 1.

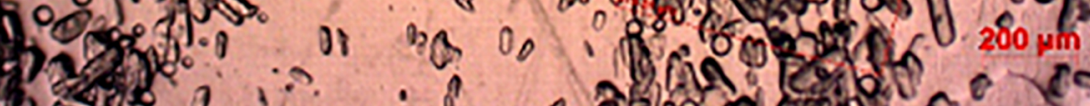

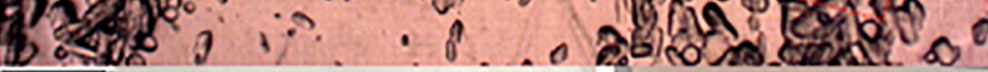


D)

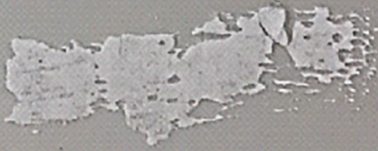

$50 \mathrm{~mm}$

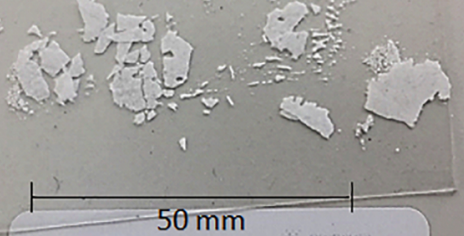

Figure 13 


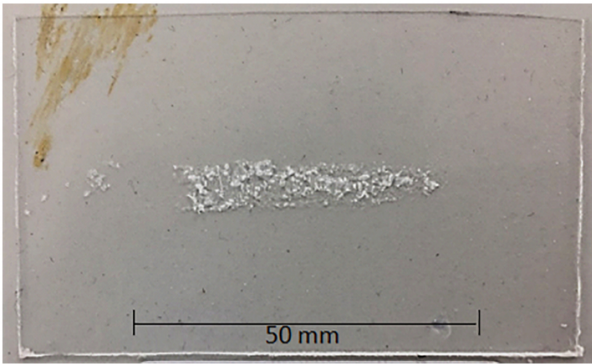

Figure 14 


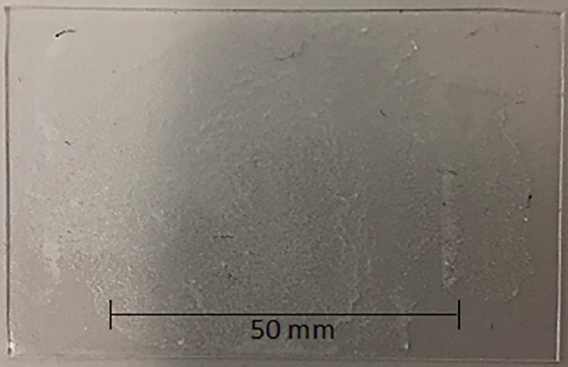

Figure 15 


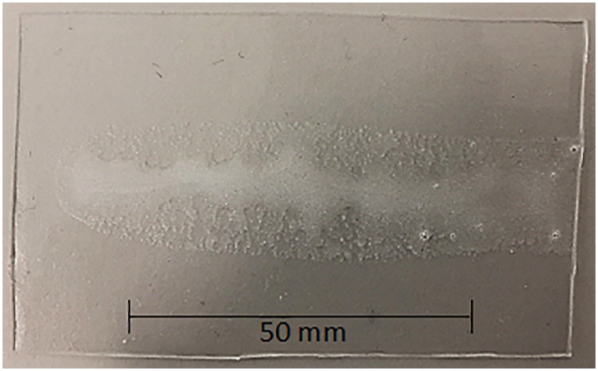

Figure 16 

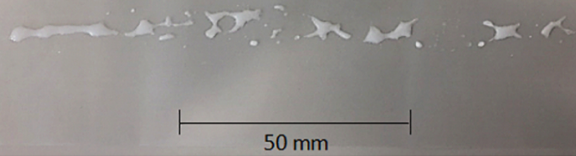

b.

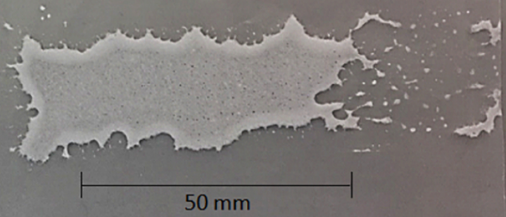

Figure 17 


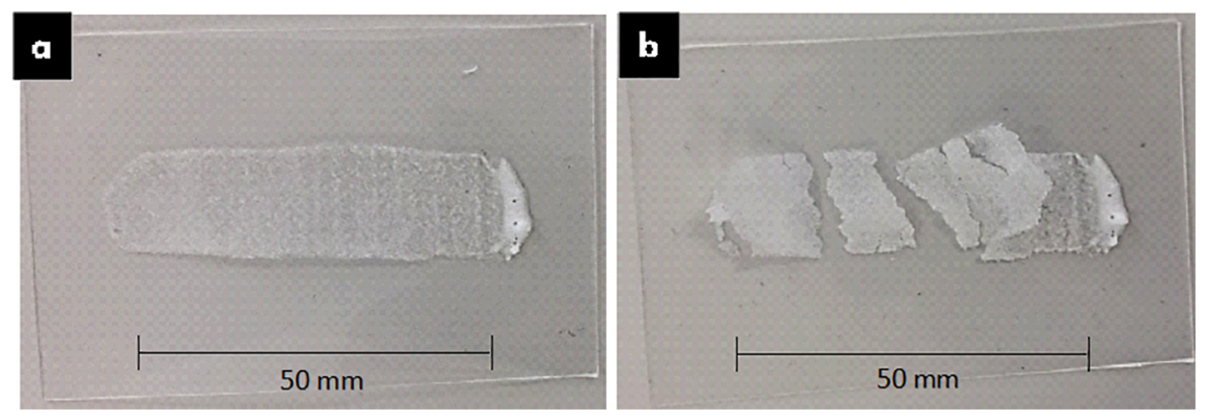

Figure 18 


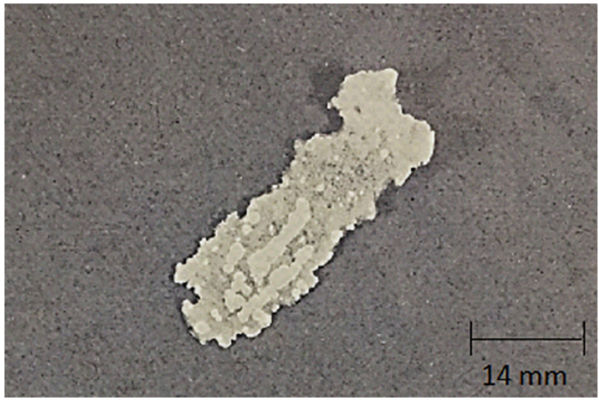

Figure 19 


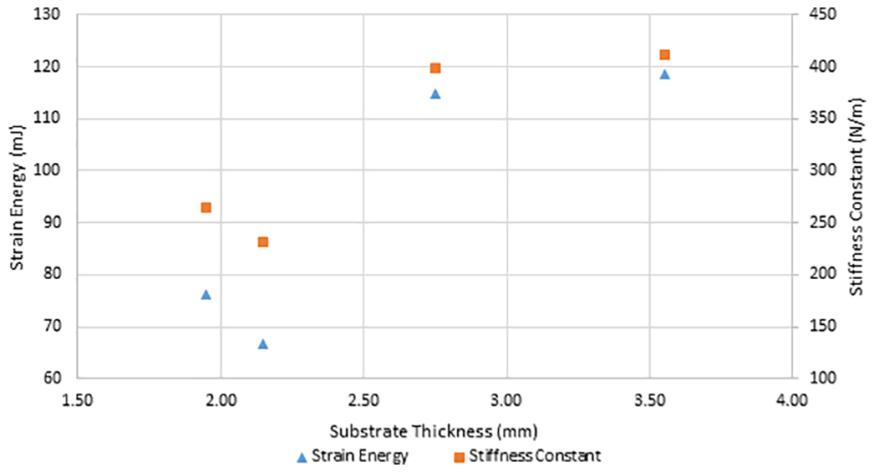

Figure 20 

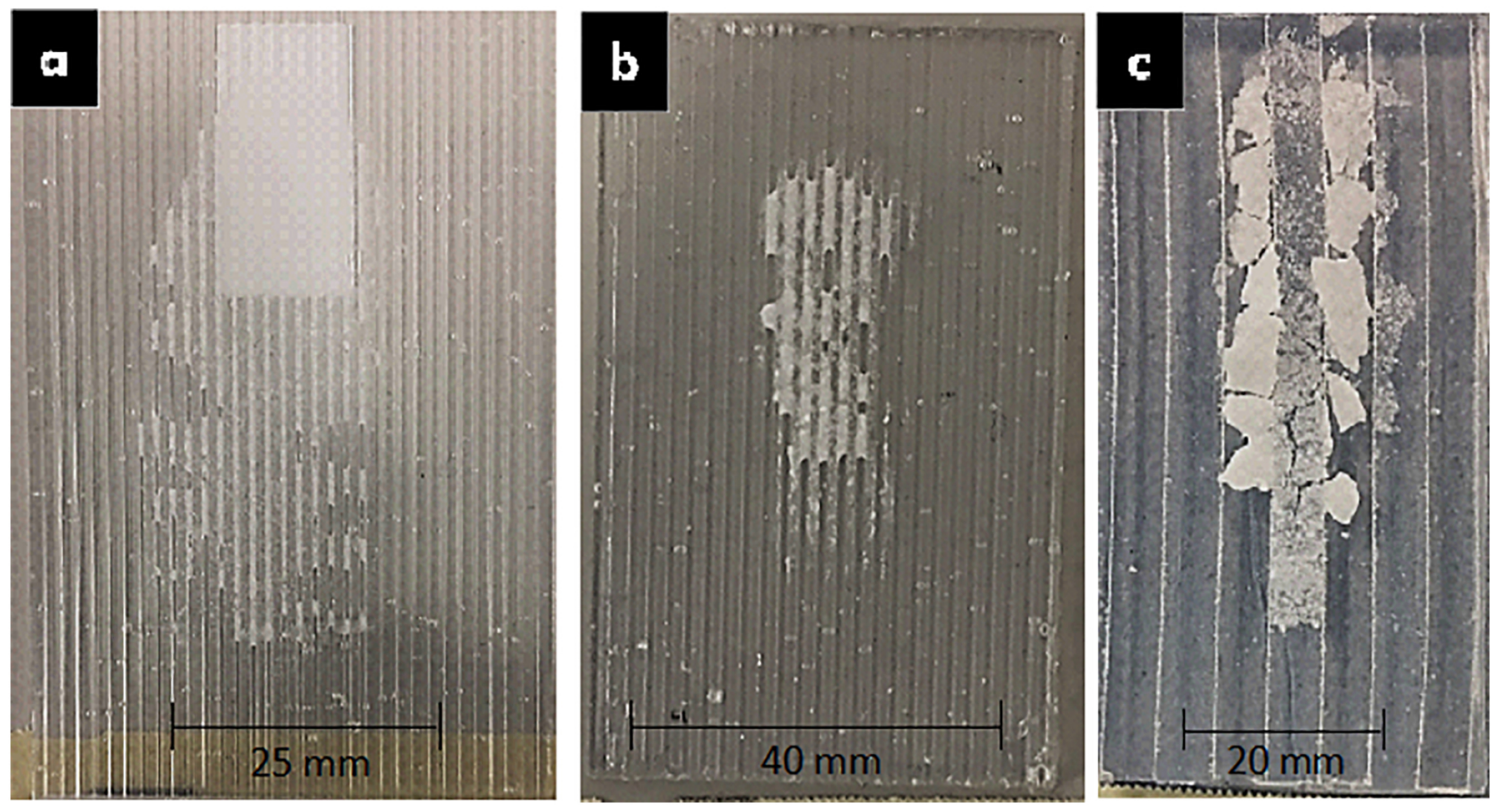

Figure 21 


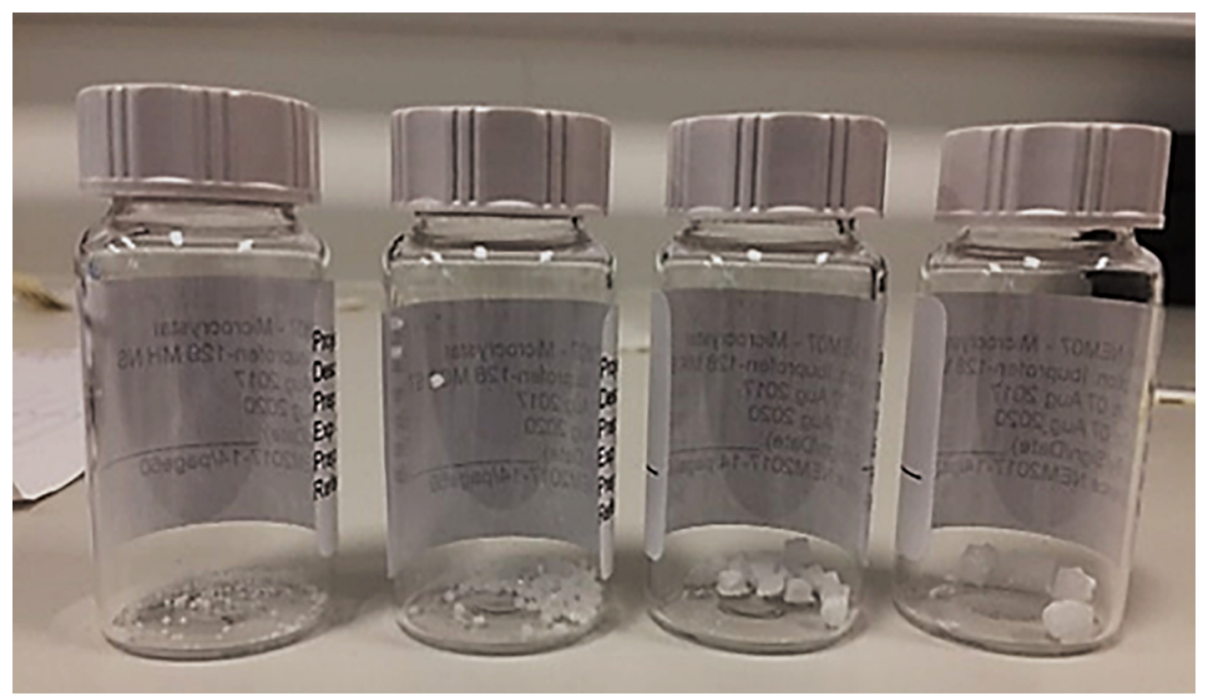

Figure 22 


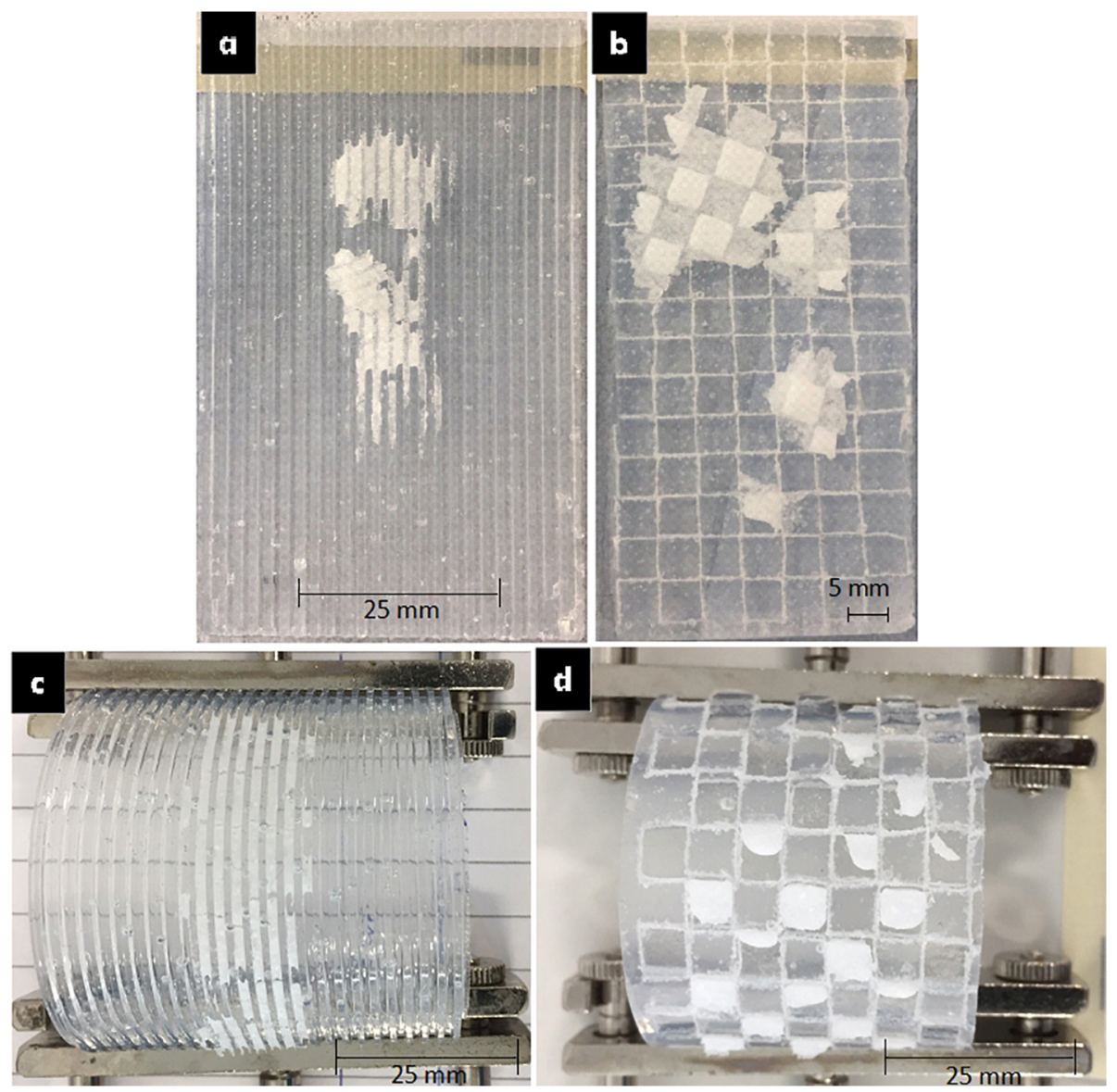

Figure 23 

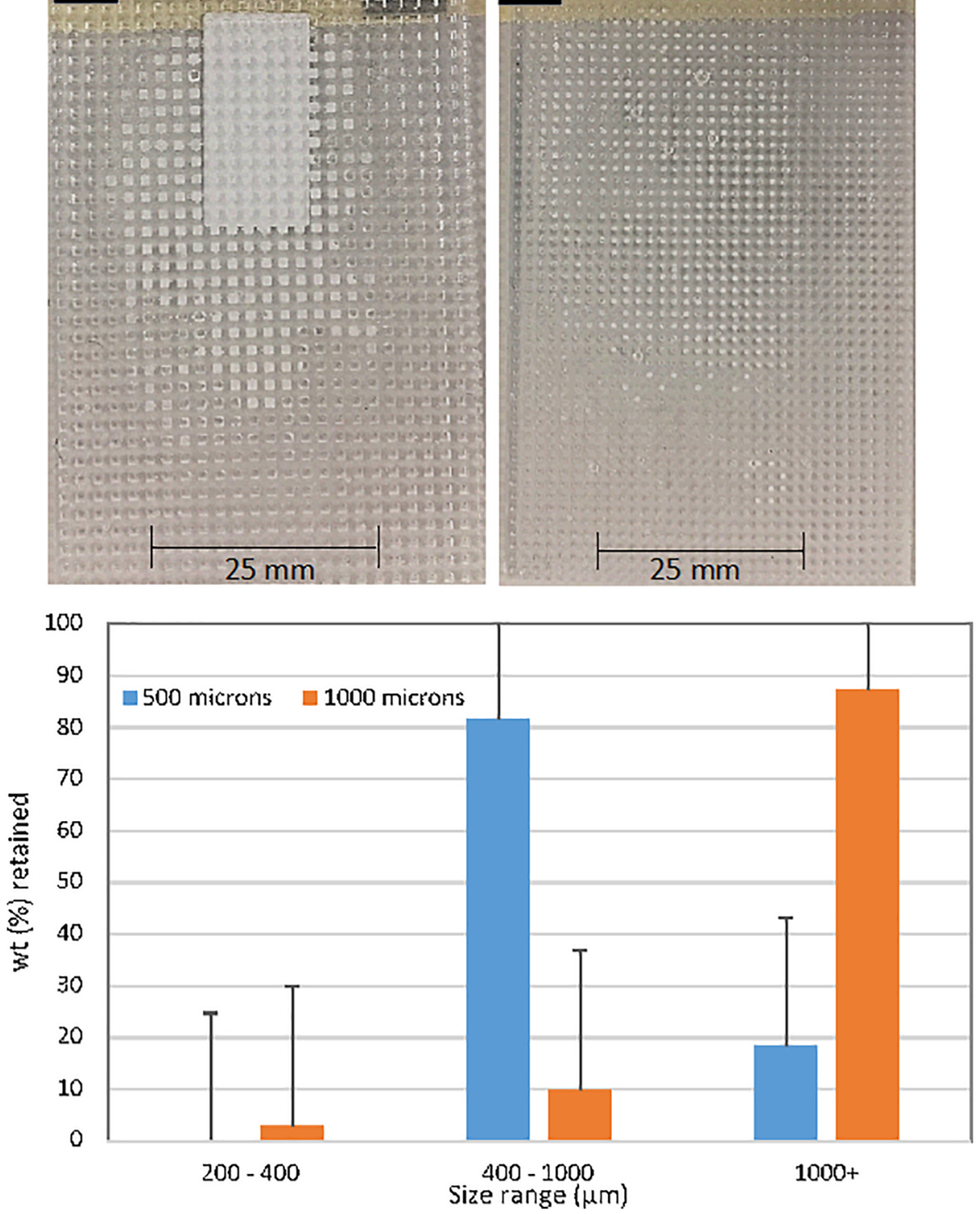

Figure 24 


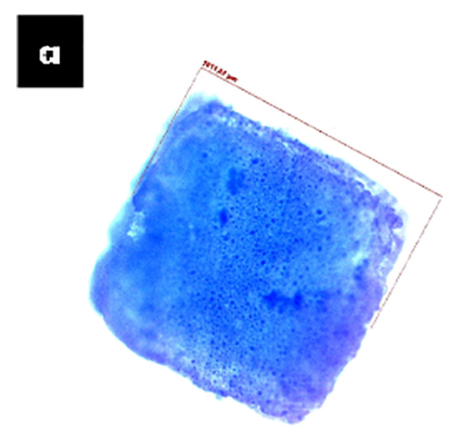

b

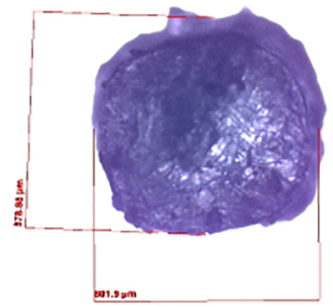

Figure 25 


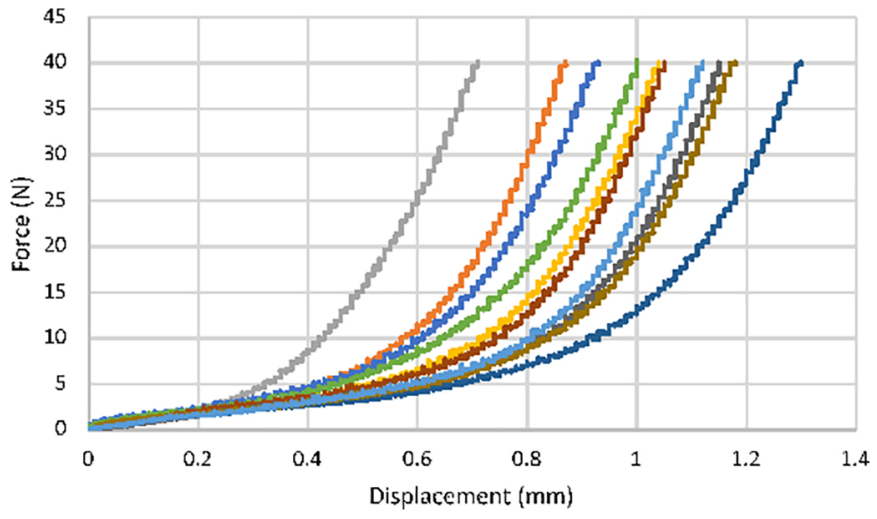

Figure 26 


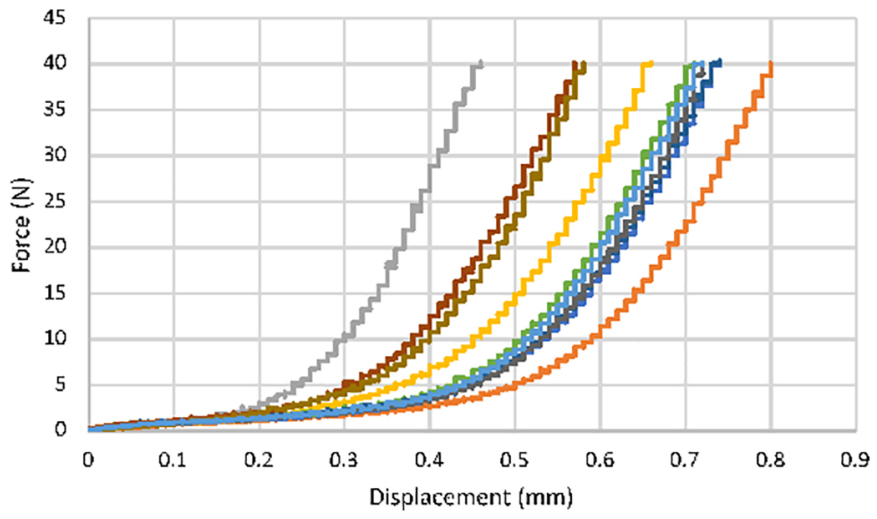

Figure 27 


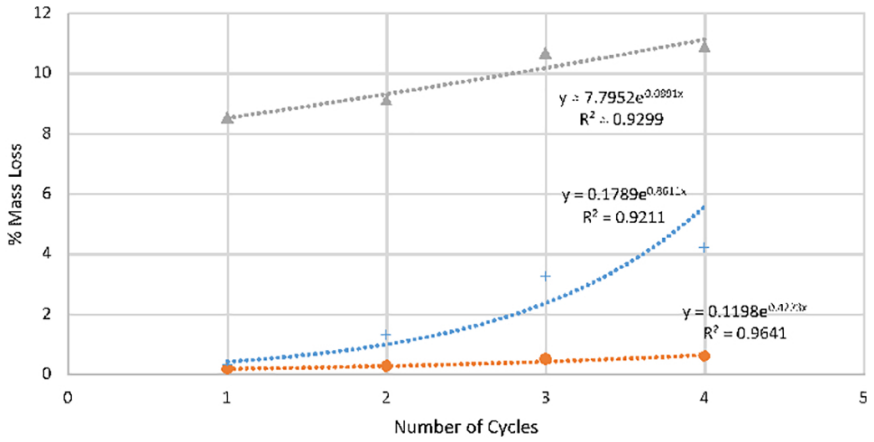

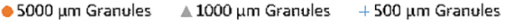

Figure 28 


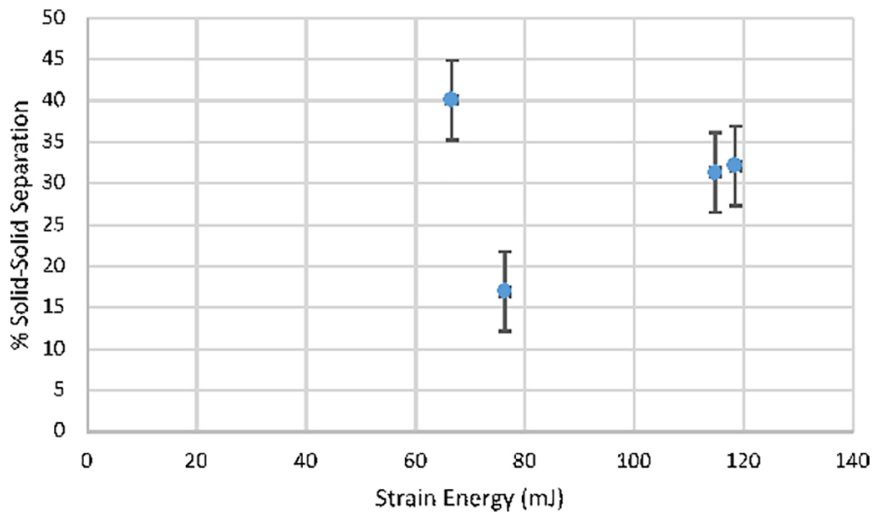

Figure 29 